rev.relac.int.estrateg.segur.5(1):119-134,2010

\title{
TERRORISMO DESMITIFICADO Verdades y Mentiras Sobre la Violencia en Colombia
}

Vicente Torrijos R.*

\section{RESUMEN}

Este es un estudio valorativo sobre la situación actual y las tendencias de las organizaciones armadas ilegales que, operando en Colombia, tienen, a nivel estratégico, una dimensión hemisférica. La conducta de tales agrupaciones es entendida aquí como una variable dependiente, condicionada por un conjunto de variables independientes específicas que se van desenvolviendo a lo largo del texto.

Palabras clave: Terrorismo, inestabilidad, riesgo, amenaza, gobernabilidad.

\section{ABSTRACT}

This is an evaluation study on the current status and trends of armed illegal organizations that, operating in Colombia, have at

\footnotetext{
* Profesor Titular de Ciencia Política y Relaciones Internacionales en la Universidad del Rosario, de Bogotá. Profesor de Lógica Estratégica en la Escuela Superior de Guerra. Consultor de medios de comunicación. Asesor para asuntos estratégicos del Comando General de las Fuerzas Militares de Colombia. tutoriascontorrijos@yahoo.com
} 
the strategic level a hemispheric dimension. The behavior of such groups is understood here as a dependent variable, conditioned by a specific set of independent variables that unfold throughout the text.

Key words: Terrorism, instability, risk, threat, gobernability

\section{Siglas}

AUC: Autodefensas Unidas de Colombia.

Bacrim: Bandas Criminales Emergentes (antiguas autodefensas).

ELN: Ejército de Liberación Nacional.

FARC: Fuerzas Armadas Revolucionarias de Colombia.

PCB: Plataforma Continental Bolivariana.

PCSD: Plan de Consolidación de la Política de Defensa y Seguridad Democrática.

PSD: Política de Defensa y Seguridad Democrática.

\section{INTRODUCCIÓN}

Hay dos axiomas fundamentales bien conocidos por los estudiosos (y los practicantes) de la lucha contrainsurgente. Según el primero de ellos, «una guerrilla gana mientras no es definitivamente derrotada, en tanto que el Estado pierde mientras no logra la victoria final». De acuerdo con el segundo, «ningún logro en la lucha contrainsurgente es absolutamente irreversible».

Al cabo de casi una década de ajustes y de reformulaciones estratégicas enmarcados en el Plan Colombia y la Política de Defensa y Seguridad Democrática (PSD), estos dos axiomas deben presidir y orientar todo esfuerzo por valorar adecuadamente y proyectar con verosimilitud los escenarios de la seguridad y la defensa en Colombia, en particular, frente a las organizaciones subversivas históricas que nacieron como guerrillas durante la segunda década del siglo pasado, vale decir, las Fuerzas Armadas Revolucionarias de Colombia (FARC) y el Ejército de Liberación Nacional (ELN) (Maullin, 1971).

Lo anterior significa reconocer que aunque estas organizaciones han venido estando sometidas a una presión militar constante que las ha obligado a replegarse estratégicamente, a rehuir la confrontación armada directa y a apelar cada vez más al uso estratégico del terrorismo, con la consecuente erosión de su cohesión interna y la capacidad de control de los órganos centrales sobre los cuadros medios periféricos, ello no significa que estén forzosamente predestinadas a la derrota, o que esta es pura cuestión de tiempo.

En efecto, organizaciones como las FARC, el ELN y hasta las Bandas Criminales Emergentes (Bacrim), antes ligadas a las fuerzas de autodefensa y hoy tan asociadas al narcotráfico, se caracterizan por 
su enorme versatilidad y resiliencia. No por esto, sin embargo, Colombia ha renunciado a la posibilidad y el desafío estratégico de derrotarlas.

\section{PRIMERA PARTE: LAS FARC Y SU CONDUCTA}

Teniendo permanentemente presente lo anterior, lo que se presenta en esta primera parte es un diagnóstico del estado actual de las FARC, en un esfuerzo por explicar sus más recientes conductas, que tal vez pudieran inscribirse en el marco estratégico delimitado por el chantaje humanitario, el terrorismo selectivo y las escaramuzas contundentes.

Tras valorar estas conductas, en la segunda parte se identificarán las variables independientes que parecen determinarla y que podrían explicar su evolución en el futuro inmediato.

\section{EL ESTADO ACTUAL DE LAS FARC}

Las FARC terminaron 2009 con un fracaso táctico que pudieron convertir en éxito estratégico (el secuestro y vil asesinato del gobernador de Caquetá, Luis Francisco Cuéllar), pues les sirvió para generar corrientes de cuestionamiento y de crítica a la PSD, a la vez que reavivó la preocupación social por el drama de los secuestrados con cuya suerte insisten en chantajear al país y acorralar al Gobierno. A su vez, el Estado ha concluido 2009 e iniciado 2010 con importantes éxitos tácticos (a pesar de los errores cometidos en el caso del gobernador), pero debe ajustar su estrategia para asegurar que los mismos tengan un impacto importante y sostenido.

Para ello es necesario responder adecuadamente a la pregunta por la situación real de las FARC, a fin de no subestimar ni sobreestimar sus actuaciones. (V. el estudio de Cragin, 2009, sobre las motivaciones de los terroristas).

\section{HIPÓTESIS CONDUCTUAL 1: PROPAGANDA MEDIANTE LA ACCIÓN}

Debilitadas en cuanto a su capacidad de despliegue armado y de combate, las FARC están encauzando (y encauzarán) todas sus energías y recursos a la ejecución de acciones de alta carga simbólica, muchas de ellas de corte terrorista, que a los menores costos les permitan cuestionar los logros alegados por el Estado en la ejecución de la PSD.

El fallido secuestro y el cruel asesinato del gobernador Cuéllar se ajusta perfectamente a esta hipótesis. Aunque el secuestro falló, pues se vieron obligados a asesinar al rehén como consecuencia de la presión de la Fuerza Pública, el incidente les sirvió para transmitir un mensaje de contundente incertidumbre y dejar una sombra de duda sobre la eficacia real de la PSD, de la que rápidamente se hicieron eco algunos analistas y comentaristas con evidente superficialidad e irresponsabilidad. 
Cabe anotar que, adicionalmente, el secuestro de Cuéllar les habría permitido replantear el tema del intercambio humanitario, incluso mientras se hacían aproximaciones con la senadora Córdoba y la Iglesia para la liberación de dos de los rehenes (que hasta ahora no se ha verificado).

\section{HIPÓTESIS CONDUCTUAL 2: INTERFERENCIA POLÍTICA}

Resulta pertinente leer la conducta de las FARC (no solo en relación con su ataque al Gobernador de Caquetá sino, por ejemplo, en relación con su «renovada alianza con el ELN» y con las escaramuzas que ha realizado en las zonas en las que aún tiene un relativo margen de actuación) en clave electoral.

Como saben que su futuro depende más de su habilidad política que de su capacidad armada, orientan su conducta en pos de interferir tanto como sea posible en el proceso electoral y las agendas de los distintos candidatos, a fin de ganar protagonismo y convertirse, por las buenas o por las malas, en árbitros del debate electoral.

La clave de esta conducta sería polarizar a la opinión en su juicio y apreciación de la PSD y forzar quizá a los candidatos a distanciarse de ella y a sugerir aproximaciones alternativas en materia de seguridad.

\section{HIPÓTESIS CONDUCTUAL 3: UNA ESTRATEGIA DE PRESTIGIO}

La conducta fariana podría interpretarse también como reflejo de una estrategia de prestigio, a través de demostraciones de fuerza, aplicada por orden del Secretariado con varios objetivos:

1. Evitar el deterioro de su imagen interna y externa frente a los éxitos de la PSD.

2. Elevar la moral de la militancia fariana.

3. Mantener un mínimo de figuración mediática que los inmunice contra el riesgo de perder el espacio ganado en el imaginario colectivo nacional.

4. Afirmar el liderazgo de «Alfonso Cano» al interior de la cúpula y controvertir la posición de sus detractores que lo acusan de debilidad.

Cierto es que esta estrategia de prestigio entrañan costos importantes, pero en el cálculo racional de las FARC, los costos de la inacción o la pérdida de protagonismo son superiores a los que estas acciones (por muy controversiales que resulten) les generan. (Cfr. el estudio de Jenkins, 2009, sobre el razonamiento estratégico de los terroristas).

\section{PRIMERA CONCLUSIÓN}

Estas hipótesis conductuales no son mutuamente excluyentes, sino que, incluso, se refuerzan recíprocamente. Por otro lado, reflejan tanto el éxito de la PSD (pues es ahora el accionar del 
Estado el que condiciona la conducta de las organizaciones armadas ilegales, que tienen que reaccionar permanentemente frente a la dinámica que este les impone), como la necesidad de reajustarla permanentemente para anticiparse a la reacción de estas organizaciones.

Las FARC parecen, en todo caso, estar sometidas a una lógica de la supervivencia. En ese sentido, están dispuestas a asumir todos los costos, como por ejemplo, aparecer con sus acciones como una organización cruel e inhumana, que no respeta las limitaciones humanitarias al uso de la fuerza, con tal de conservar (y demostrar) un mínimo de prestigio y de capacidad de lucha. La coyuntura interna (de contienda electoral) e internacional, se muestra además sumamente propicia para desplegar este tipo de demostraciones.

\section{SEGUNDA PARTE: LA EVOLUCIÓN DE LA CONDUCTA FARIANA}

A lo largo de 2009, y en perfecta consonancia con el hecho innegable de que la implementación de la PSD y sus estrategias de consolidación (PCSD) han impactado y deteriorado sustancialmente su capacidad ofensiva y operativa, la actividad de las FARC parece haberse enmarcado en función de tres hipótesis conductuales:

1. El permanente recurso a la propaganda mediante la acción.

2. La búsqueda constante de oportunidades de interferencia en el proceso político por vías diversas (no exclusivamente armadas, no necesariamente directas).

3. El mantenimiento de un grado mínimo de actividad en desarrollo de una estrategia de prestigio que le provea de un espacio vital básico, tanto en términos políticos como militares.

La conducta fariana, que puede interpretarse con arreglo a estas tres hipótesis conductuales, puede considerarse a su vez como una variable dependiente que evoluciona, se proyecta y se contrae en función de un conjunto de factores que operan como variables independientes y que, con toda seguridad, continuarán influyendo notablemente en el comportamiento de esa organización en el mediano plazo.

Esta segunda parte identifica y valora sintéticamente seis de esas variables, que también podrían servir como indicadores del riesgo de fortalecimiento o re-empoderamiento de la organización y que, por lo tanto, pueden funcionar como insumos óptimos para la planeación de la capacidad de respuesta y anticipación del Estado colombiano frente al desafío narcoterrorista que esta encarna. (Johnson, 2008, provee elementos valiosos para entender mejor esta dinámica de reforzamiento de capacidades, aún cuando se trate de un fenómeno meramente aparente). 


\section{VARIABLE 1. APOYO DIRECTO O INDIRECTO DE UNA POTENCIA (PEQUEÑA, MEDIANA O GRANDE), YA SEA REGIONAL O EXTRA-REGIONAL.}

Con o sin el apoyo material, simbólico y político efectivo del régimen chavista y su proyecto expansionista de «revolución bolivariana» y "socialismo del siglo XXI», resulta innegable que la suerte y el comportamiento de Venezuela influyen significativamente en el comportamiento fariano.

Esta influencia se puede identificar en varios niveles:

1. Como referente ideológico y empírico (y por lo tanto, inspirador) de una forma política alternativa de carácter revolucionario, del cual han carecido las FARC durante muchos años, pero que ahora parece encarnar (con alcance continental) el Alba-Chavismo.

2. Como interlocutor bien dispuesto y, por lo tanto, como eventual validador internacional de algunas iniciativas políticas de las FARC.

3. Como «amparo», en la medida en que el Alba-Chavismo actúe con lasitud o negligencia en el control fronterizo, la lucha contra el narcotráfico o, yendo más lejos, proporcione santuario real y efectivo a las actividades y cabecillas de la organización.

4. Como dispositivo perturbador de las relaciones bilaterales y regionales y, por lo tanto, como factor de perturbación de la gobernabilidad interna del país (mediante la polarización frente a temas clave, como la cooperación con los Estados Unidos). (Un marco comprehensivo para explicar las relaciones de cooperación militar entre Colombia y los Estados Unidos puede verse en Kelly, 2009).

Uno de los recursos para canalizar y operativizar esta influencia es sin duda la llamada «Plataforma Continental Bolivariana» (PCB). Aunque su potencial y su capacidad de acción no debe sobreestimarse, resulta innegable que a través de la misma el chavismo «blanquea» sus simpatías hacia las FARC, y estas «allanan» su propio acceso a algunos escenarios políticos internacionales, amplían su interlocución con actores transnacionales y encuentran una óptima red para la difusión de su propaganda tendenciosa.

\section{VARIABLE 2. INTENSA FUNDAMENTACIÓN Y PROPAGACIÓN IDEOLÓGICA.}

Debilitadas militarmente, las FARC buscan afanosamente vigorizar y renovar su discurso político y su penetración social. Sus vínculos con la PCB y sus enlaces con algunos sectores políticos en Colombia son elementos clave en ese proceso, tanto más cuanto que tienen en la mira el proceso electoral de 2010, en el que, si bien saben que será muy difícil (prácticamente imposible) derrotar el Uribismo, pueden llegar a posicionar en el debate algunos temas clave como el del intercambio humanitario, como solución al drama del secuestro, o el de la necesidad de reintentar (en su propio beneficio, claro está) una solución negociada a la confrontación con el Estado. (Cfr. el conjunto de referentes históricos que presenta Schultz, 1969). 
Hay que destacar, por lo tanto, que las FARC han venido refinando y reforzando su agenda y sus objetivos políticos. Que saben que no pueden derrotar electoralmente al Uribismo, pero que pueden erosionar la gobernabilidad, y que pueden para ello instrumentalizar tanto la sensibilidad de la opinión frente a algunos temas como el legítimo interés de la oposición democrática de cuestionar las políticas del gobierno, sin descartar, eso sí, el activo trabajo de sus infiltrados en esa oposición y en otras instancias sociales.

\section{VARIABLE 3. GRADO RELATIVO DE APOYO POPULAR A LA CAUSA REVOLUCIONARIA, Y GRADO DE VERSATILIDAD ORGANIZACIONAL.}

Ninguna insurgencia sobrevive sin un apoyo mínimo de la población. Así, las FARC se han venido concentrando en capitalizar ciertas fuentes de apoyo históricas: el campesinado cocalero, los contrabandistas de combustibles, etc, pero también, las comunidades (especialmente rurales) en las que la militancia fariana tiene un profundo anclaje histórico y transgeneracional (en zonas como Cauca, Nariño y Tolima grande).

Ello no les ha impedido buscar nuevos espacios de colonización ideológica, en una labor que podría intensificarse de cara al escenario electoral de 2010, con la aspiración a movilizar a la población en pro de ciertos candidatos con quienes pudiera tener (sistemáticamente) o encontrar (coyunturalmente) alguna coincidencia.

\section{VARIABLE 4. CAPITALIZACIÓN DE EMERGENCIAS SOCIALES Y POLÍTICAS COMPLEJAS.}

El déficit social acumulado en el país, y la polarización política inducida, principalmente por la ambigua posición del presidente Uribe frente a su aspiración a una nueva reelección, pueden convertirse en hontanar de la movilización y el posicionamiento político de las FARC en el mediano plazo.

Por un lado, están todavía por verse las consecuencias de la crisis financiera en el país, y es necesario encontrar la forma de resolver importantes demandas en política social, cuya atención se ha visto limitada como consecuencia de la necesidad de hacer mayores esfuerzos presupuestales orientados al sector defensa.

Por el otro, con todas las ventajas que una eventual reelección del presidente Uribe pudiera traer consigo, vienen también los costos y los riesgos asociados: fragmentación de las élites, disminución de la gobernabilidad congresional, intensificación del enfrentamiento entre poderes y, sobre todo, la posibilidad de que en ese escenario el propio presidente Uribe aparezca como el artífice de un paulatino desmonte de la Constitución de 1991 y de una nueva re-concentración del poder en el ejecutivo. 
En esas condiciones, las FARC intentarán capitalizar los descontentos (políticos y sociales), sobre todo para desacreditar interna y externamente al gobierno, y para cuestionar su legitimidad. Romper el consenso y dificultar la tarea de gobernar pueden ser para las FARC objetivos tan deseables como un despeje o la toma de una capital de departamento. (Cfr. antecedentes interesantes en Slighton, 1968).

\section{VARIABLE 5. DIVERSIFICACIÓN RENTABLE Y PERDURABLE DE FUENTES DE FINANCIACIÓN.}

Las FARC siguen enfrentando el problema de cómo convertir sus activos (resultado de la capitalización del narcotráfico) en insumos efectivos para la operación militar y el sostenimiento logístico de sus unidades de combate. (V. los interesantes hallazgos sobre la relación entre terrorismo y drogas, en Reuter, 2009).

Este problema les ha impedido resolver la disparidad tecnológica frente a las Fuerzas Militares; y al mismo tiempo, impacta en su cohesión interna, por cuanto hay varios frentes sometidos a una importante presión de sostenibilidad financiera.

En la perspectiva de que no puedan resolverlas en el corto plazo, estas constricciones seguirán limitando su capacidad operativa, por lo cual tenderán a eludirlas intensificando su actividad terrorista e intimidatoria, mediante ataques a objetivos puntuales o mediante una reactivación de la práctica del secuestro (empleado como táctica de impacto comunicacional, principalmente).

En efecto, este factor limita enormemente su capacidad de aprovechar las ventajas que podrían derivar de su asimetría para lanzarse a una guerra irregular como la que pudieron desplegar en el pasado, a menos, claro, que un actor externo intensificara sustancialmente su apoyo facilitándoles el acceso a los recursos de los que requeriría lanzarse nuevamente a la ofensiva.

\section{VARIABLE 6. CAPITALIZACIÓN DE UNA DOMINACIÓN POLÍTICA DESPÓTICA, OCUPACIÓN EXTRANJERA O INTERVENCIONISMO.}

Aunque Colombia no está ocupada por ninguna potencia extranjera, ni está siendo intervenida desde el exterior (pues la ayuda externa que recibe tanto de Europa como de los EE UU es consensuada, concertada y coordinada), la subversión podría aprovechar, tanto política como tácticamente, la intensificación de la cooperación con los Estados Unidos en el marco del Acuerdo Complementario de Cooperación suscrito con ese país el año pasado. (Drezner, 2009, presenta un interesante marco de análisis sobre la innovación estratégica en un marco de complejidad).

El contexto informacional y el debate regional que ha suscitado el acuerdo, así como la polarización que generó en el mundo político interno, podrían ser capitalizados por las FARC a través de operaciones selectivas de impacto contra objetivos norteamericanos, o de las cuales se pudiera 
derivar un refuerzo para el argumento de que el Gobierno colombiano está abriendo las puertas a una «intervención imperialista» en el país.

\section{SEGUNDA CONCLUSIÓN}

Hasta ahora se han identificado algunos vectores que determinan la dinámica de la seguridad y la defensa en Colombia actualmente, así como tres hipótesis conductuales de las FARC.

A este esquema de análisis lo complementan las 6 variables que dan cuenta tanto de debilidades como de fortalezas y oportunidades que podrían ser aprovechadas, por una parte, para neutralizar y anticipar la acción de las FARC, y por otra, por esa misma organización, para apuntalar su estrategia de supervivencia y perturbación de baja intensidad, aún en las difíciles condiciones operativas en las que se encuentran como consecuencia del exitoso despliegue de la PSD durante los últimos siete años.

\section{TERCERA PARTE: EL ELN Y SU VOLUNTAD DE LUCHA}

A pesar de su aparente debilidad, el Ejército de Liberación Nacional ha demostrado ser una de las organizaciones armadas ilegales con mayor capacidad de resiliencia (es decir, de recuperación tras enfrentar experiencias traumáticas), adaptabilidad (acomodamiento a circunstancias y entornos cambiantes y adversos) y redundancia (capacidad de transferir el desgaste de uno de sus componentes a los otros mediante un delicado sistema de compensación).

Durante los últimos 8 años el ELN, en efecto, dejó hundir la posibilidad de alcanzar una negociación con el Estado colombiano al abandonar el dispendioso proceso de «encuentros exploratorios» justo cuando parecía estar por fin afinado un Acuerdo Base que serviría de puntal para una interlocución integral y definitiva que condujera a la desmovilización de la organización.

Durante los últimos 8 años, también, el ELN ha sufrido el impacto de la Política de Defensa y Seguridad Democrática (PSD) que, sin Ilegar a erradicarlo, lo ha diezmado por lo menos, habida cuenta de su menor densidad y de su menor disponibilidad y acceso a recursos en comparación, por ejemplo, con las FARC.

No obstante, el ELN está hoy todavía lejos de haber sido definitivamente derrotado, y aunque su actividad ha disminuido sustancialmente, no debe ser subestimado como amenaza real para la seguridad en Colombia.

Ello es así en buena medida por cuanto el ELN, quizá en parte por pura inercia organizacional, parece conservar su voluntad de lucha, es decir, su decisión de persistir en la confrontación con el Estado por la vía militar o, por lo menos, en la expectativa de provocar y propiciar condiciones 
favorables a la misma, tanto mediante el uso táctico del terrorismo como de la movilización y la agitación de las masas. Lo anterior, claro está, con independencia de cualquier probabilidad realista de éxito en el presente.

Durante 2009, en total fueron dejados «fuera de combate», bien por capturas, muerte en combate o entregas voluntarias de sometimiento a las autoridades, un total de 505 miembros del ELN, que entre tanto concentró su accionar en zonas de Arauca, Chocó, Valle, Cauca y Nariño, muchas veces en confrontación y otras en connivencia con otras organizaciones ilegales.

En términos generales, la conducta y contumacia del ELN parecen explicarse según un conjunto de estrategias de conservación, orientadas a mantener a la organización con vida y con un mínimo grado de actividad, mientras espera un escenario más favorable para recuperar la iniciativa y el impulso dinámico que le permita relanzarse a la confrontación masiva y directa con el Estado. Este conjunto de estrategias se analiza sucintamente a continuación.

\section{ESTRATEGIA DE CONSERVACIÓN 1: SIMBIOSIS PARASITARIA}

Para compensar su debilidad estructural y organizacional, y mantenerse con vida a pesar de la inactividad forzosa y el permanente acorralamiento por parte de la Fuerza Pública, el ELN apela cada vez con más frecuencia a lo que pudiera denominarse «simbiosis parasitaria», en la que actúa esencialmente como parásito, pero al mismo tiempo beneficia a su huésped.

La mejor expresión de esta estrategia es la «alianza» genérica establecida con las Fuerzas Armadas Revolucionarias de Colombia (FARC), que fuera anunciada por ambas organizaciones a finales del año 2009. (Esta situación puede llevar al Estado a ciertas inconsistencias estratégicas coyunturales, como puede apreciarse en Robinson, 2010).

No debe descartarse que acuerdos semejantes, aunque de menor impacto político y mucho más pragmáticos, existan con algunas bandas criminales en ciertas zonas del país.

Y está por demás decir que estas relaciones simbiótico-parasitarias no excluyen la competencia armada con otros grupos, especialmente por el control de algunos corredores vitales del negocio de las drogas.

\section{ESTRATEGIA DE CONSERVACIÓN 2: RELAJAMIENTO}

Debilitado en cuanto a su capacidad de despliegue armado y de combate, el ELN viene sufriendo también una creciente pérdida de cohesión interna, que se traduce en una disminución de la efectiva capacidad de control de las instancias superiores (el Comando Central) sobre las unidades inferiores. 
A fin de impedir el desvertebramiento masivo de la organización, como consecuencia de las fuerzas centrífugas liberadas por la pérdida de cohesión, la organización parece haber optado por relajar el control centralizado y ampliar el margen de maniobra autónoma de estas unidades, lo cual, además, le permite transferirles la responsabilidad por su supervivencia y sostenibilidad, de las cuales ya no puede ser garante ni árbitro absoluto el mando central.

\section{ESTRATEGIA DE CONSERVACIÓN 3: REFUGIO}

Para preservar la línea de mando, esencial para su figuración política, y consciente del impacto que tendría en la organización un golpe similar al sufrido por las FARC con la «Operación Fénix» (en marzo de 2008, sobre territorio ecuatoriano), el ELN ha decidido apelar al refugio de sus cabecillas en el santuario que le ofrecen algunas locaciones en el exterior (en particular, y muy probablemente, en Venezuela).

Esta estrategia de refugio quizá complica los canales de comunicación y dirección inmediata de la organización, pero ese precio parece compensarlo la garantía de conservación y resguardo de la dirigencia, que mientras tanto intensifica su actividad política y propagandística a nivel internacional.

\section{ESTRATEGIA DE CONSERVACIÓN 4: FOCALIZACIÓN Y PERTURBACIÓN}

La conducta del ELN, en estas circunstancias, está determinada en buena medida por la necesidad de ahorrar recursos y energía, preservarse del desgaste y maximizar el impacto de sus acciones.

De ahí que la focalización de las mismas constituya la clave de su apuesta por la perturbación.

Esto explica la concentración de su actividad en áreas de dominio histórico (como Arauca), en las que a su conocimiento acumulado del entorno se añade, como ventaja operativa, la existencia de una base social de afiliación transgeneracional (esto es, la sucesión de una generación a otra de la militancia en la organización).

También se refleja en el desplazamiento o incremento de sus actividades a áreas anteriormente copadas por las autodefensas ilegales y todavía sólo parcialmente recuperadas por el Estado.

Y desde luego, ya no en función de la perturbación sino de la supervivencia de la organización, explica la intensificación de su actividad (en competencia con otros grupos armados ilegales y organizaciones criminales) en las zonas neurálgicas de actividades macrodelictivas, en especial, el narcotráfico.

\section{ESTRATEGIA DE CONSERVACIÓN 5: DEPREDACIÓN}

Esto último refleja una final estratégica de conservación: el incremento de la actividad puramente depredatoria de los recursos naturales y de la actividad económica (lícita e ilícita). 
Esta estrategia se ciñe a una lógica esencialmente económica, pero sigue estando subordinada al propósito político fundamental de la organización, que ahora mismo no es otro que el de su autopreservación.

En ese sentido, se explica la intensificación de las actividades criminales del ELN de corte extractivo de la riqueza (vía narcotráfico, por ejemplo) en algunas zonas y, eventualmente, podría traducirse en un renacimiento de prácticas tradicionales de financiamiento de la organización de carácter puramente extorsivo.

\section{TERCERA CONCLUSIÓN}

Las estrategias aquí identificadas tienen un carácter eminentemente hipotético, pero pueden ser empleadas como insumos útiles en la construcción de escenarios y la valoración de potenciales frente al devenir futuro del ELN. (Treverton, 2009, ilustra bastante bien sobre las exigencias que un desafío como este supone en materia de inteligencia estratégica).

Por lo tanto, a pesar de sus limitaciones, contribuyen a identificar las vulnerabilidades específicas de la organización en el actual escenario de la seguridad en Colombia, concentrándose en los aspectos particularmente significativos que han ido estableciendo un orden estratégico de prioridades con el que se ha cerrado progresivamente el cerco sobre la organización ilegal.

\section{CUARTA PARTE: LA SOBERBIA DE LAS BANDAS CRIMINALES EMERGENTES}

Al desafío en materia de seguridad que siguen encarnando organizaciones armadas ilegales de origen insurgente como las FARC y el ELN, cabe añadir el fenómeno reciente, aunque no sin precedentes en la historia del país, de las Ilamadas «Bandas Criminales Emergentes» (Bacrim) que operan en diversas regiones.

Estas Bacrim son fundamentalmente organizaciones criminales (macro-delincuenciales) significativamente armadas, que desarrollan actividades tanto de control de grandes negocios ilícitos como de depredación subsidiara de los mismos, y que con frecuencia emplean la violencia como mecanismo de disciplinamiento interno, de delimitación de áreas de influencia específicas y de coacción e intimidación unilateral sobre terceros a fin de mantener las condiciones de operación requeridas por sus actividades. (Cfr. la valoración de potenciales que en el caso asiático hace Chalk, 2009).

A diferencia de las tradicionales organizaciones armadas ilegales de origen insurgente, las Bacrim carecen por completo de cualquier plataforma (así sea puramente formal y retórica) y objetivo (plausible o no) de carácter político. 
En ese sentido, son esencialmente manifestaciones de criminalidad organizada (en tanto que organizaciones estables y permanentes, estructuradas funcionalmente, cuyo propósito es la ejecución de uno o más delitos graves a efectos de proporcionar a sus integrantes un volumen creciente de beneficios económicos).

Ello explica por qué son sustancialmente refractarias a todo tipo de incentivo o constricción de carácter político, formuladas indirectamente por actores sociales como la Iglesia Católica. Ofertas de sometimiento, rebaja de penas y reintegración pasan a ser, por lo tanto, inocuas como mecanismo para reducirlas y desarticularlas.

Es necesario también distinguir estas Bacrim de las antiguas organizaciones de autodefensa ilegal, a pesar de que en algunos medios se insista en que son simplemente un reencauche o reedición de aquellas en lo que se ha venido a Ilamar «neoparamilitarismo».

Las antiguas autodefensas ilegales tenían un propósito contrainsurgente, del que hoy por hoy carecen las Bacrim. Por otro lado, el hecho de que estas bandas hayan surgido en zonas antaño bajo influencia de las autodefensas ilegales, de que se hayan articulado con base en estructuras también heredadas de aquellas, y de que algunos de sus miembros hayan estado previamente vinculados a las autodefensas ilegales, no hace de ellas una mera prolongación del viejo «paramilitarismo».

Lo anterior no implica desconocer que, sin embargo, las mismas estructuras sociopolíticas y económicas (ligadas sobre todo al narcotráfico y a la fragilidad de las instituciones y la democracia locales), que se convirtieron en oportunidad para el auge y expansión de las autodefensas ilegales, también han contribuido a la aparición de las Bacrim y a alimentarla con los insumos (materiales e institucionales) de los que se alimentaron los paramilitares y con los reductos (humanos) de estas organizaciones no absorbidos por el proceso de Justicia y Paz.

\section{CARACTERIZACIÓN DEL FENÓMENO}

Carentes de todo motor de naturaleza política, estas bandas tienden a comportarse según el clásico patrón de la macrodelincuencia organizada, también en cuanto tiene que ver con el alcance de su uso de la violencia como con su relacionamiento con el poder político y económico a nivel local. (Cfr. los interesantes aportes sobre tráfico de armas de Cragin \& Hoffman, 2003).

No se puede decir que se trate de grupos sustitutos y continuadores de las Autodefensas Unidas de Colombia (AUC), sólo que atomizados y fragmentados. Y esto a pesar de que en muchos casos los líderes de las nuevas formaciones sean mandos medios provenientes de las AUC, actúen en las mismas zonas y operen de manera similar (control territorial + coacción + control poblacional + narcotráfico). 
En resumen, aunque se trate de fenómenos asociados, autodefensas ilegales y Bacrim no pueden confundirse.

\section{FACTORES DE OPORTUNIDAD}

Con todo, la evidencia de algunas coincidencias entre las Bacrim y las AUC debe ser valorada como insumo útil para evaluar el proceso de sometimiento y desmovilización de estas últimas organizaciones, a fin de evitar que en ocasiones futuras se repitan las mismas falencias y, por decir algo, así como hoy existen Bacrim asociadas a reductos no sometidos de las AUC, se generen en el futuro Bacrim asociadas a reductos no sometidos o eficientemente desmovilizados de las FARC o el ELN.

Dentro de los factores de oportunidad más importantes para la formación de estas Bacrim (en relación con el «legado» de las AUC) pueden señalarse:

La persistencia de los lazos y redes entre integrantes de antiguos bloques y unidades de las AUC, que no fueron disueltos a través, por ejemplo, de su dispersión geográfica como condición de desmovilización (en el caso de los llamados «reincidentes»).

- Las dificultades jurídicas y policiales para identificar y extinguir el dominio sobre los bienes de muchos de los mandos medios de estas organizaciones.

- La persistencia de lazos con las autoridades locales, en una especie de versión «micro» de la denominada "parapolítica», que debe ser todavía investigada.

\section{IMPLICACIONES EN MATERIA DE SEGURIDAD}

En buena medida, la actividad de las Bacrim está asociada al incremento de los índices de criminalidad y, en particular, de homicidio, registrados en algunas zonas del país.

En todo caso, fenómenos como el de las Bacrim son, por demás, habituales en el marco de procesos de paz, y es verdad sabida que luego de todo proceso de desmovilización se presenta la tendencia de algunos sectores de estas organizaciones a proseguir con el uso de la violencia, con arreglo a una dinámica básicamente criminal y lucrativa.

Todo lo anterior implica un nuevo panorama en materia de seguridad: la amenaza que representan las Bacrim es básicamente un problema policial, y así lo ha entendido el Estado colombiano.

Pero no se puede subestimar el potencial desestabilizador, más allá de lo puramente policial, de su capacidad armada y del conocimiento acumulado y heredado de las antiguas AUC.

Si existe algún modelo de amenaza híbrida, quizá el de las Bacrim pueda ser un buen prototipo de ello y, por lo tanto, para enfrentarla se barajan también estrategias híbridas, centradas en su carácter 
delincuencial, pero sin desconocer su enorme capacidad de perturbación social e incluso de control territorial, derivada del copioso volumen de recursos que manejan y de su conocimiento y acceso a un armamento más sofisticado y poderoso del que normalmente tienen los grupos criminales clásicos.

\section{CUARTA CONCLUSIÓN}

La aparición de bandas criminales era previsible consecuencia del proceso de sometimiento de las AUC. En ese sentido, la experiencia está siendo aprovechada para incorporar un aprendizaje que en el futuro minimice la repetición de ese riesgo, por ejemplo, en un escenario de desmovilización masiva de otras organizaciones armadas ilegales.

Por otro lado, aunque no se las puede considerar meras continuadoras o reciclaje de las AUC, debe admitirse que han medrado aprovechando las mismas estructuras de oportunidad que antaño facilitaron la expansión de las AUC y, por lo tanto, su neutralización efectiva habría que buscarse en la eliminación radical de dichas estructuras. (Gompert, 2009, plantea, justamente, una serie de elementos que podrían tenerse en cuenta bajo la noción de reconstrucción social desde el conflicto). Por último, su naturaleza híbrida, criminal y armada, plantea un reto en materia de seguridad que no se restringe solo al ámbito policial (aunque sea este el abordaje más ajustado y pertinente), sino que implica diseñar un modelo igualmente híbrido y complejo para contenerlas y erradicarlas, o para que gradualmente entiendan la necesidad de someterse a la justicia sin ningún tipo de recompensas o prebendas.

\section{BIBLIOGRAFÍA}

- $\quad$ CHALK, P.; Rabasa, A. Rosenau, W. \& Leanne Piggott (2009) The Evolving Terrorist Threat to Southeast Asia. A Net Assessment. Rand Corporation,Sta. Mónica.

- $\quad$ CRAGIN, K. (2009) Understanding Terrorist Motivations. Rand Corporation, Sta. Mónica.

- $\quad$ CRAGIN, K. \& Hoffman, B. (2003) Arms Trafficking and Colombia. Rand Corporation, Sta. Mónica.

- $\quad$ DREZNER, J. A. (2009) Competition and Innovation Under Complexity. Rand Corporation, Sta. Mónica.

- $\quad$ GOMPERT, D. C.; Kelly, T. K.; Lawson, B. S.; Parker, M. \& Colloton, K. (2009) Reconstruction Under Fire. Unifying Civil and Military Counterinsurgency. Rand Corporation, Sta. Mónica.

- $\quad$ JENKINS, B. M. (2009) Terrorists Can Think Strategically. Lessons Learned From the Mumbai Attacks. Rand Corporation, Sta. Mónica. 
- $\quad$ JOHNSON, D. E.; Grissom, A. \& Oliker, O. (2008) In the Middle of the Fight. An Assessment of Medium-Armored Forces in Past Military Operations. Rand Corporation, Sta. Mónica.

- $\quad$ KELLY, T. K. ; Jones, S. G. ; Barnett, J. E. ; Crane, K. ; Davis, R. C. \& Jensen, C. (2009) Does the United States Need a New Police Force for Stability Operations? Rand Corporation, Sta. Mónica.

- $\quad$ MAULliN, R. L. (1971) Soldiers, Guerrillas, and Politics in Colombia. Rand Corporation, Sta. Mónica.

- $\quad$ REUTER, P. H. ; Trautmann, F. ; Pacula, R. L. ; Kilmer, B. ; Gageldonk, A. \& Gouwe D. (2009) Assessing Changes in Global Drug Problems, 1998-2007. Rand Corporation, Sta. Mónica.

- $\quad$ ROBINSON, N.; Potoglou, D.; Kim, Ch. W.; Burge, P. \& Warnes R. (2010) Security, At What Cost? Quantifying people's trade-offs across liberty, privacy and security. Rand Corporation, Sta. Mónica.

- $\quad$ SCHULTZ, T. P. (1969) Internal Migration. : A Quantitative Study of Rural-Urban Migration in Colombia. Rand Corporation, Sta. Mónica.

- $\quad$ SLIGHTON, R. L. (1968) Relative Wages, Skill Shortages, and Changes in Income Distribution in Colombia. Rand Corporation, Sta. Mónica.

- $\quad$ TREVERTON, G. F. (2009) Intelligence for an Age of Terror. Rand Corporation, Sta. Mónica. 